\title{
AN ANALYTICAL APPROACH TO THE POISSON EQUATION IN 3-DIMENSIONAL SPACE CHARGE PROBLEMS
}

\author{
S. Valero*, P. Lapostolle**, A.M. Lombardi***, N.Pichoff*, E. Tanke*** \\ * CEA-DSM-SEA, Saclay, F-91191 Gif-sur-Yvette Cedex, France \\ ** Consultant, PS Division, CERN, CH-1211 Geneva 23, Switzerland \\ *** PS Division, CERN, CH-1211 Geneva 23, Switzerland
}

\begin{abstract}
The problem of electrostatics repulsion between charged particles in a bunch is a classical mixed DirichletNeumann problem. In this paper, an analytical solution of this problem is described. The approach proposed here can be extended to other problems in mathematical physics.
\end{abstract}

\section{INTRODUCTION}

In an accelerator a bunched beam consists of an arbitrary system of charged particles embedded in a finite volume. The surface separating this system of particles from the environment possesses often a complicate form difficult to define. Moreover the density (number of particles per unity of volume) does not generally follow the usual mathematical statistics laws. However the forces acting on the particles are well-known and the behaviour of the system of particles can be in principle deduced from its distribution. knowing the position of these particles, the effects of the electrostatic repulsion between the particles can be calculated. The problem could be simplified in finding appropriate solutions of the Poisson equation $\Delta U=-\rho / \varepsilon_{0}$ where $\rho$ is the electric charge density, and $U$ the electrostatic potential.

\section{THE ELECTROSTATIC PROBLEM OF THE BUNCH}

Summarising the more general problem in electrostatics for a system of particles embedded in a finite volume $\mathrm{V}$, one obtains a mixed Dirichlet-Neumann problem. The solution of this problem requires the conditions prescribed on the boundary $\mathrm{S}$ of a finite region within $\mathrm{V}$. When the system of charged particles is arbitrary, these conditions cannot be easily defined, and one substitutes the following problem:

i) $\quad \Delta U=-\rho / \varepsilon_{0}$

ii) $\quad \mathrm{U}$ and its partial derivatives are null at $\infty$ (1)

iii) $\quad \rho$ is continuously null outside a finite region

The density $\rho$ will be obtained from the system of charged particles. For a system of particles lying in a closed volume, this problem can be considered identical to the Dirichlet-Neumann problem[1].

\section{BEST-APPROXIMATION OF THE DENSITY FUNCTION WITH 3-D SERIES}

The density function must be expressed in a suitable analytical form, allowing to compute the potential. To do so, the density is expanded in a series of 3-dimensional orthogonal functions. As the limits of the system of particles are not well-known, only 3-d orthogonal functions requiring no strict boundary limits must be retained. Periodic orthogonal functions (as Fourier series, for example) are complicated since they introduce image effects resulting of the periodicity. A good candidate, satisfying the conditions $\rho=0$ at, $\infty$ can be obtained by a generalisation in 3-d of the Hermite functions.

One defines :

$\delta_{l m n}(x, y, z)=\Psi_{l}(x) \Psi_{m}(y) \Psi_{n}(z)$,

where $\Psi_{l}(x)$ are the Hermite orthogonal functions generated from the following defining relation :

$\Psi_{l}(x)=(-1)^{l} d^{l}\left(e^{-x^{2} / 2}\right) / d x^{l}=H_{l}(x) e^{-x^{2} / 2}$,

where $H_{l}\left(x_{i}\right)$ are the orthogonal Hermite polynomials $[2,4]$ of degree 1 , forming a complete sequence of orthogonal functions in the functional space $L^{2}\left(R^{3}\right)$ of the functions measurable with the following Lebesgues-measure [3]:

$\mathrm{d} \Phi=\mathrm{e}^{\left(\mathrm{x}^{2}+\mathrm{y}^{2}+\mathrm{z}^{2}\right) / 2} \mathrm{dxdydz}$.

One endows this functional space with a scalar product :

$<\mathrm{f}, \mathrm{g}>=\iiint_{\mathrm{R}^{3}} \mathrm{f}(\mathrm{x}, \mathrm{y}, \mathrm{z}) \mathrm{g}(\mathrm{x}, \mathrm{y}, \mathrm{z}) \mathrm{d} \Phi$.

The density $\rho$, being null outside a finite region, belongs to this functional space. One can then expand the density in the complete basis:

$\rho(x, y, z)=\sum_{l=0}^{\infty} \sum_{m=0}^{\infty} \sum_{n=0}^{\infty} \frac{\left.<\rho, \delta_{l m n}\right\rangle}{\left.<\delta_{l m n}, \delta_{l m n}\right\rangle} \delta_{l m n}(x, y, z)$

Let's consider now a finite sequence $\left\{\delta_{\mathrm{Imn}}\right\}$ with $0 \leq l, m, n \leq l_{1}, m_{1}, n_{1}$. This sequence generates a functional space $\mathrm{X}\left(\mathrm{l}_{1}, \mathrm{~m}_{1}, \mathrm{n}_{1}\right)$ endowed with the scalar product defined in Eq. 5. The finite sequence $\left\{\delta_{\mathrm{Imn}}\right\}$ constitutes a complete basis of orthogonal functions, and any function in this space can be expanded in this basis. One can prove [3] that there exists one element and only one element $S_{1}(\rho)$ in $\mathrm{X}\left(\mathrm{l}_{1}, \mathrm{~m}_{1}, \mathrm{n}_{1}\right)$, such that the following distance: 


$$
D=\left|\iiint_{R^{3}}\left(\rho(x, y, z)-S_{1}(\rho)\right) d x d y d z\right|
$$

is minimum. This element is the projection of the density $\rho$ in the functional space $X(11, m 1, n 1)$. Expanding this projection with the basis one obtains :

$$
S_{1}(\rho)=\sum_{l=0}^{l_{1}} \sum_{m=0}^{m_{1}} \sum_{n=0}^{n_{1}} \frac{<\rho, \delta_{l m n}>}{<\delta_{l m n}, \delta_{l m n}>} \delta_{l m n}(x, y, z) \text {. }
$$

One can show [3] that, contemplating a system of $\mathrm{N}$ charged particles, one has :

$$
\frac{<\rho, \delta_{l m n}>}{<\delta_{l m n}, \delta_{l m n}>}=\frac{1}{(2 \pi)^{3 / 2} l ! m ! n !} \sum_{i=1}^{N} H_{l}\left(x_{i}\right) H_{m}\left(y_{i}\right) H_{n}\left(z_{i}\right),
$$

where $\mathrm{x}_{\mathrm{i}}, \mathrm{y}_{\mathrm{i}}$, and $\mathrm{z}_{\mathrm{i}}$ are the co-ordinates of the particles.

One also proves $[2,3]$ that the distance D given in Eq.7 is bounded, and one has :

$$
\mathrm{D} \leq \mathrm{C} \frac{\mathrm{l}_{1} ! \mathrm{m}_{1} ! \mathrm{n}_{1}}{\left(2 \mathrm{l}_{1}\right) !\left(2 \mathrm{~m}_{1}\right) !\left(2 \mathrm{n}_{1}\right) !},
$$

where $\mathrm{C}$ is a constant depending of the density.

The general expression of the field components is given by :

$\mathrm{E}_{\mathrm{x}}(\mathrm{x}, \mathrm{y}, \mathrm{z})=\frac{1}{\varepsilon_{0}} \iiint_{\mathrm{R}^{3}} \frac{\xi \rho(\mathrm{x}+\xi, \mathrm{y}+\mu, \mathrm{z}+\varsigma)}{\left(\xi^{2}+\mu^{2}+\varsigma^{2}\right)^{3 / 2}} \mathrm{~d} \xi \mathrm{d} \mu \mathrm{d} \zeta$.

The density $\rho$ being positive or null in a finite region, from the first theorem of the average[4], the truncation error introduced in the potential or in the field components, when the density $\rho$ is replaced by $S_{1}(\rho)$, could be deduced from the distance $\mathrm{D}$.

\section{ANALYTICAL SOLUTION OF THE POISSON EQUATION}

In the problem (1), the density $\rho$ can be replaced by it's approximation $S_{1}(\rho)$ and one considers separately each term in the series in Eq. (8) :

$$
\Delta U^{*}(u, v, w)=-\frac{1<\rho, \delta_{l m n}>}{\varepsilon_{0}<\delta_{l m n}, \delta_{l m n}>} \delta_{l m n}(u, v, w) .
$$

These $\mathrm{u}, \mathrm{v}$ and $\mathrm{w}$ are the normalised co-ordinates obtained in scaling the co-ordinates along the principal axes relative to the r.m.s. dimensions $\mathrm{a}, \mathrm{b}$ and $\mathrm{c}$ of the bunch. Applying a Fourier transformation to Eq. (12), one obtains an expression of the field component :

$$
\begin{aligned}
& E_{x}^{*}(u, v, w)=(-i)^{l+m+n+1}(2 \pi)^{-3 / 2} \frac{<\rho, \delta_{l m n}>}{\varepsilon_{0}<\delta_{l m n}, \delta_{l m n}>} \\
& \times \int_{-\infty}^{\infty} \int_{-\infty}^{\infty} \int_{-\infty}^{\infty} \frac{\hat{u}^{l+1} \hat{v}^{m} \hat{w}^{n}}{\frac{\hat{u}^{2}}{a^{2}}+\frac{\hat{v}^{2}}{b^{2}}+\frac{\hat{w}^{2}}{c^{2}}} e^{\frac{\hat{u}^{2}+\hat{v}^{2}+\hat{w}^{2}}{2}} e^{i(\hat{u} u+\hat{v} v+\hat{w} w)} d \hat{u} d \hat{v} d \hat{w} .
\end{aligned}
$$

An analytical solution of this 3-d complex integral can be found by separating it into three 1-d complex integrals. This process leads to very sizeable analytical calculations [3], whom one only gives here the principle. One considers for instance in the 3-d integral above, the following integral function :

$$
I_{1}(\hat{v}, \hat{w})=a^{2} \int_{-\infty}^{\infty} \frac{\hat{u}^{l+1}}{\hat{u}^{2}+\frac{a^{2}}{b^{2}} \hat{v}^{2}+\frac{a^{2}}{c^{2}} \hat{w}^{2}} e^{-\hat{u}^{2} / 2} e^{i \hat{u} u} d \hat{u} .
$$

From the Cauchy residue theorem and from an integral representation of the probability function, one obtains an analytical expression of $I_{1}(\hat{v}, \hat{w})$ in terms of Hermite functions. Introducing this expression in the 3-d integral in Eq. (13), one has to calculate a new integral function :

$I_{2}(\hat{w})=\int_{-\infty}^{\infty} \hat{v}^{m} e^{-\hat{v}^{2} / 2} I_{1}(\hat{v}, \hat{w}) e^{i \hat{v} v} d \hat{v}$.

The Cauchy residue theorem and the properties of the Hermite functions, allow to obtain an other analytical expression in terms of Hermite functions. This expression introduced in Eq. (13), enable to obtain an analytical expression of the 3-d integral.

Further, in the aim to save computer time, integrals above could be calculated through expansions around specific positions in the bunch.

\section{EXAMPLES}

Figures 1-5 illustrate an estimation of the influence of the truncation error, done when the density $\rho$ is replaced by its truncated series developments defined in Eq. (8), on the potential or on the field components calculated with the same 3-d Gauss numerical integration on a sphere. This numerical integration has been used in place of the analytical method explained in this paper, because this can only be applied to the series developments. The "noise" observed on the curves is induced by the numerical integration.

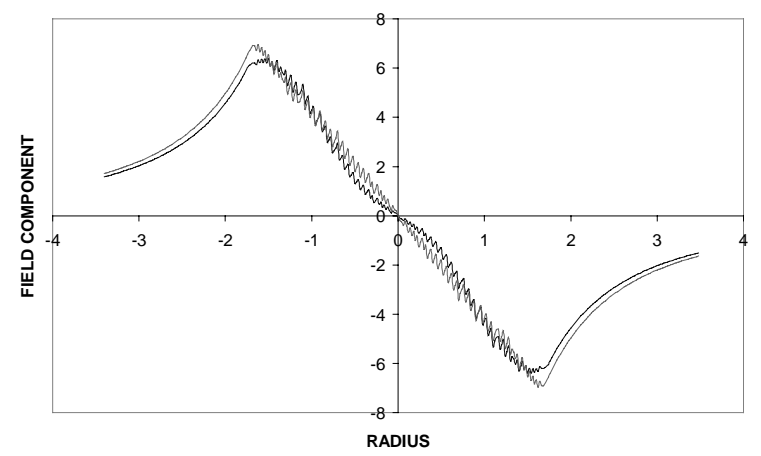

Figure 1: The radial field component along a diameter of a sphere for which the density $\rho(x, y, z)=1$ is compared with the same component as calculated by series developments with $\mathrm{l}_{1}, \mathrm{~m}_{1}$ and $\mathrm{n}_{1}=12$. Due to the symmetry, only the terms in the series with even $1, \mathrm{~m}$ and $\mathrm{n}$ are not null. 


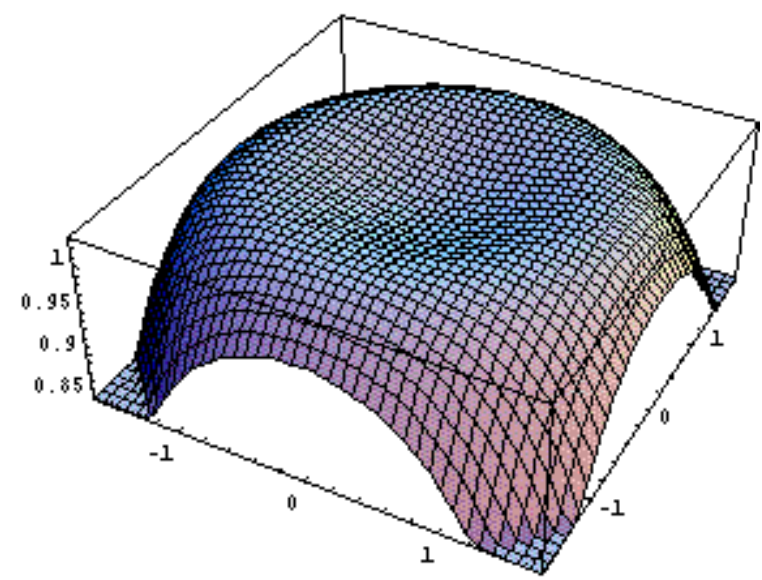

Figure 2: The density is a $4^{\text {th }}$ order spherical function, represented here at $\mathrm{z}=0$.

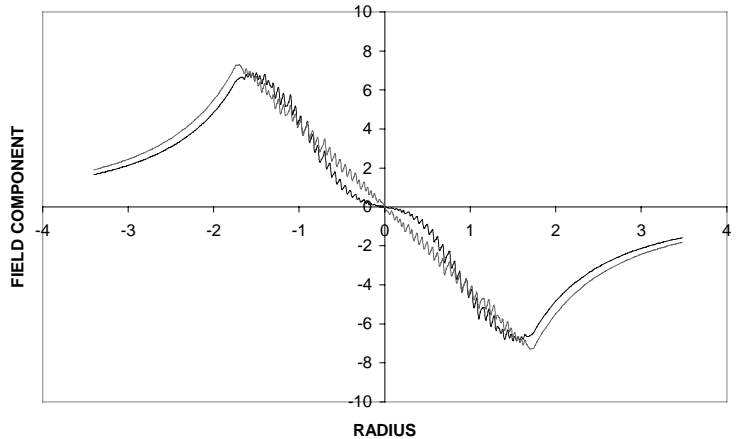

Figure 3: The field component $\mathrm{E}_{\mathrm{x}}$ deduced from the density given in fig. 2 is compared with this obtained from the truncated series with $\mathrm{l}_{1}, \mathrm{~m}_{1}$ and $\mathrm{n}_{1}=6$. The difference vanishes when increasing this values to 12 (see fig. 1).

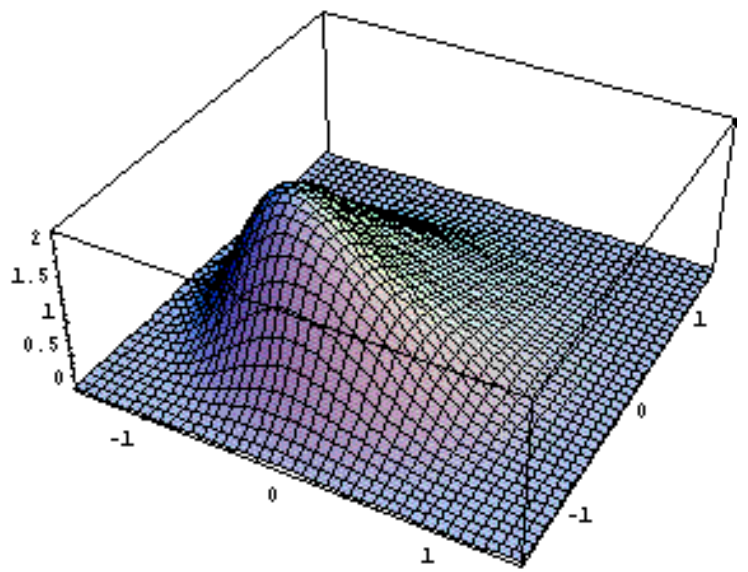

Figure 4: The density is a non-symmetrical function represented here at $\mathrm{z}=0$.

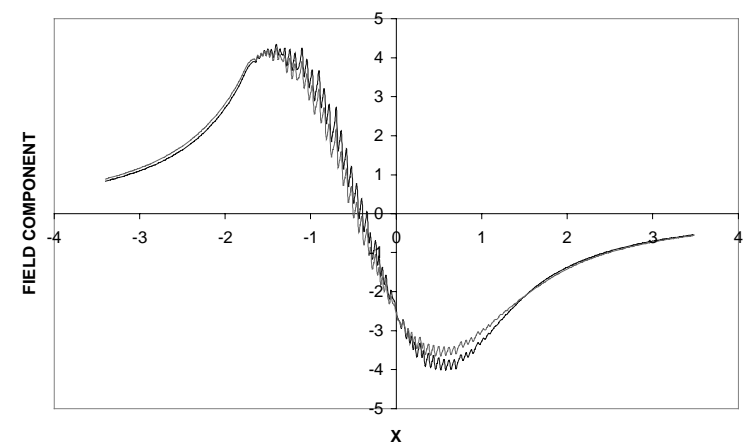

Figure 5: Field component $\mathrm{E}_{\mathrm{x}}$ along $\mathrm{x}$-axis with a beam density given in fig.4, compared with this calculated from series developments with $\mathrm{l}_{1}, \mathrm{~m}_{1}$ and $\mathrm{n}_{1}=7$. Here the terms in the series, with odd and even $1, \mathrm{~m}$ and $\mathrm{n}$ are used. As in the practical cases, the density is here continuous at the boundary of its defining area, contrary to previous example (fig.2).

\section{CONCLUSION}

The present work suggest a new type of approach leading to an analytical solution of a classical Dirichlet-Neumann problem in 3-dimensions. It is particularly suitable for an arbitrary system of charged particles without symmetry, when the limits of the system are not well-known. It can be extended to other problems in mathematical physics. A new routine is being developed solve space-charge effects in accelerators. It could become a good tool helping in the estimation of tolerances necessary for the design and operation of high intensity linacs. Some possible refinements of the present method are still being studied. This process might also be used for cyclotrons.

\section{ACKNOWLEDGMENTS}

We thank B. Aune and J. M. Lagniel from Saclay and H. Haseroth and W. Pirkl from CERN for their interest and support.

\section{REFERENCES}

[1] Ian Sneddon. Elements of Partial Differential Equations, Chapter 4. McGraw-Hill Kogakusha (1957).

[2] A.M. Cohen. Numerical Analysis, Chapter 1,2. McCraw-Hill book Co., (1973).New York

[3] An Analytical Approach to the Solution of the Poisson Equation in 3-dimentional Electrostatics Problems. To be published by the authors.

[4] G.Valiron. Théorie des Fonctions, Chapitre IV. Masson et $C^{\text {ie }}$ Paris(1955). 